\title{
Three Dimensional Measurements Of Geodesic Acoustic Mode with Correlation Doppler Reflectometers
}

\author{
W.L. Zhong ${ }^{* 1}$, Z.B. Shi ${ }^{1}$,Y. Xu ${ }^{1}$, X.L. Zou ${ }^{2}$, X.R. Duan ${ }^{1}$, W. Chen ${ }^{1}$, M. Jiang ${ }^{1}$, Z.C. \\ Yang $^{3}$, B.Y. Zhang ${ }^{1}$, P.W. Shi ${ }^{1}$, Z.T. Liu ${ }^{1}$, M. Xu ${ }^{1}$, X.M. Song ${ }^{1}$, X.T. Ding ${ }^{1}$, J.Q. Dong ${ }^{1,4}$, \\ Yong Liu' ${ }^{1}$ and HL-2A team ${ }^{1}$ \\ ${ }^{1}$ Southwestern Institute of Physics, P.O. Box 432, Chengdu 610041, China \\ ${ }^{2}$ CEA, IRFM, F-13108 Saint-Paul-lez-Durance, France \\ ${ }^{3}$ School of Physics and Chemistry, Xihua University, Chengdu, People's Republic of China \\ ${ }^{4}$ Institute for Fusion Theory and Simulation, Zhejiang University, China \\ E-mail: zhongwleswip.ac.cn, shizbeswip.ac.cn, xuyh@swip.ac.cn, \\ xiao-lan.zoudcea.fr, duanxr@swip.ac.cn, chenw@swip.ac.cn, \\ jiangm@swip.ac.cn, yangzc@swip.ac.cn, zhangby@swip.ac.cn, \\ shipweswip.ac.cn, liuzhetian@swip.ac.cn, minxulswip.ac.cn, \\ songxmeswip.ac.cn, dingxteswip.ac.cn, jiaqieswip.ac.cn, \\ liuyong@swip.ac.cn
}

\begin{abstract}
Correlation Doppler reflectometers have been newly developed in the HL-2A Tokamak. Owing to the flexibility of the diagnostic arrangements, the multi-channel systems allow us to study, simultaneously, the radial properties of edge turbulence and its long-range correlation in both the poloidal and toroidal direction. With correlation Doppler reflectometers, three-dimensional spatial structure of Geodesic Acoustic Mode (GAM) is surveyed, including the symmetric feature of poloidal and toroidal $E_{r}$ fluctuations, and the radial propagation of GAMs. The bi-coherence analysis for the $E_{r}$ fluctuations suggests that the three-wave nonlinear interaction could be the mechanism for the generation of GAM.The temporal behavior of GAM during the plasma density modulation experiments has been studied. The results show that the collisional damping plays a role in suppressing the GAM magnitudes, and hence, weakening the regulating effects of GAM on ambient turbulence. Three dimensional correlation Doppler measurements of GAM activity demonstrate that the newly developed correlation Doppler reflectometers in HL-2A are powerful tools for edge turbulence studies with high reliability.
\end{abstract}

1st EPS conference on Plasma Diagnostics

14-17 April 2015

Frascati, Italy

${ }^{*}$ Speaker. 


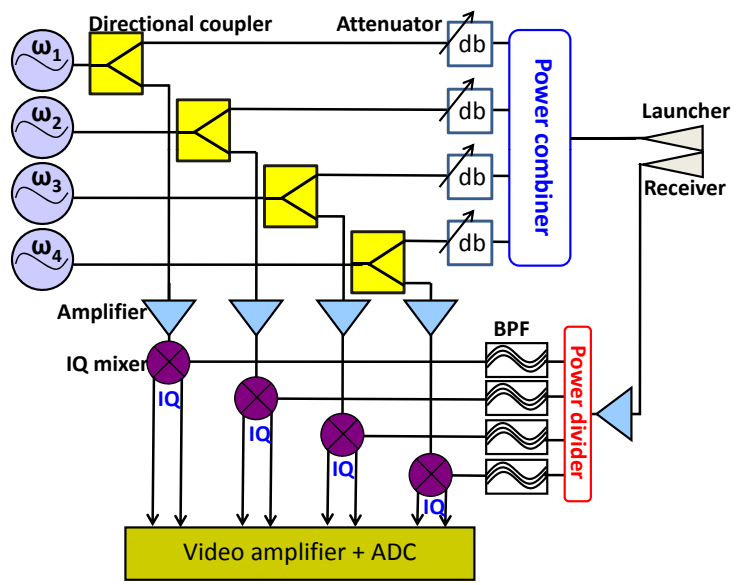

Figure 1: A schematic diagram of the multi-channel Doppler reflectometry on HL-2A.

\section{Introduction}

Doppler reflectometry was dedicated to measure the perpendicular rotation velocity of plasma turbulence and the density fluctuation simultaneously in magnetically confined plasmas $[1,2,3]$. Hence, this diagnostic can measure the radial electric field $\left(E_{r}\right)$ and its fluctuations with high spatiotemporal resolution. Thus, it is a powerful tool for the study of Zonal flow, which has a $E_{r}$ perturbation radially localized in toroidal plasmas $[4,5,6]$. Because of the flexibility of the diagnostic arrangements, the spatiotemporal characteristics of Zonal flows were obtained in both L-mode and H-mode plasmas [7, 8, 9, 10], even the spatial distribution of GAM in density fluctuation was evidenced by poloidal correlation reflectometry [11].

One of the main differences between conventional or radial reflectometry and Doppler reflectometry is the tilt angle of the launched microwave beam relative to the normal of the cut-off layer. The principle can be derived from the the energy and momentum conservation laws for coherent microwave scattering [3]. According to the Bragg selection condition, the scattered wave vector is $k_{s}=2 k_{i} \sin \left(\theta_{s}\right)$, where $k_{i}$ is the incident wave vector and $\theta_{s}$ is the tilt angle. If the turbulence moves in poloidal direction, a Doppler shift $f_{D}$ is induced in the frequency domain of the scattered wave and is determined by $f_{D}=V_{\perp} 2 k_{i} \sin \left(\theta_{s}\right) / 2 \pi$, where $V_{\perp}$ is the perpendicular velocity of turbulence. Then radial electric field $E_{r}$ can be revealed from $E_{r}=-V_{E \times B} B$ ( $B$ is the local magnetic field) by ignoring the phase velocity of the edge drift wave turbulence[7]. Hence, $E_{r}$ is proportional to the Doppler shift $\left(E_{r}=-\frac{2 \pi f_{D}}{2 k_{i} \sin \left(\theta_{s}\right)} B\right)$. With the Doppler reflectometry, the mean and fluctuant $E_{r}$ can be measured routinely. In this paper, the diagnostic setup and data processing methods for $E_{r}$ fluctuations study will be introduced firstly. Then the investigation of GAM characteristics will be presented.

\section{Diagnostic setup and data processing methods}

Recently, the microwave reflectometers, especially the Doppler reflectometers have been developed in the HL-2A tokamak [12], whose major and minor radius are $R=1.65 \mathrm{~m}$ and $a=0.4 \mathrm{~m}$ 
[13]. The multi-channel systems are dedicated to routinely measure the turbulence with high spatiotemporal resolution not only in the edge but also in the core of plasma. The microwave is generated from a novel filter-based feedback loop microwave source, which has low phase noise[14]. Figure 1 illustrates the schematic diagram of the Doppler reflectometry, which is a homodyne system. Each multi-channel system has four microwave frequencies with the spacing of $2 \mathrm{GHz}$. And the four frequencies are combined in a low insertion loss multiplexer $(<2 d B)$. The combined microwave with output power $>+15 \mathrm{dBm}$ is launched into the target plasma. Then, the four filtered waves from the power divider are separately demodulated with the reference waves in the microwave I/Q mixers. In HL-2A, there are several ports available for the arrangement of Doppler reflectometers in different poloidal and toroidal locations. Figure 2a shows the poloidal arrangement of two systems, both can operate as extraordinary mode (X-mode) and ordinary mode (O-mode) polarization. For the study of GAM in the discharge with low toroidal magnetic field $\left(B_{t}=1.3 T\right)$, the X-mode polarization is set for the multi-channel Doppler reflectometers with launching frequencies of $32,34,36$ and $38 \mathrm{GHz}$. Here, the sensitive perpendicular wave-number of the density fluctuation is $k_{\perp} \sim 3.5-5.5 \mathrm{~cm}^{-1}$ as predicted by ray tracing calculation, and the density profile is measured by the frequency modulated continuous wave reflectometry [15].

As mentioned above, $E_{r}$ is proportional to the Doppler frequency shift $f_{D}$. Generally, the time evolution of $f_{D}$ is extracted by the Centre of Gravity (CoG) of a double-sided Doppler spectrum, which given by a sliding Fast Fourier Transform (FFT) of the complex amplitude signal from the I/Q detector [7]. It is derived by $f_{D}(t)=\frac{\int f S(f, t)}{\int S(f, t)}$. Another commonly used method is to estimate the instantaneous frequency from a phase derivative (the phase is obtained by $\phi(t)=$ $\tan ^{-1}\{Q(t) / I(t)\}$ from the In-phase $(I=A \cos \phi)$ and Quadrature $(Q=A \sin \phi)$ fluctuation signals). It is given by $f_{D}(t)=\frac{1}{2 \pi} \frac{d \phi}{d t}=\frac{1}{2 \pi} \frac{\phi(t+\triangle t)-\phi(t)}{\Delta t}$. In CoG method, the equivalent sampling frequency of $f_{D}$ is determined by the interval of two sliding FFT realizations. While, it is determined by $\triangle t$ in the method of phase derivative. Fig.2 shows the compared $f_{D}(t)$ by using these two methods. The radial position of the measurement is about $4 \mathrm{~cm}$ inside the LCFS. Here, an Ohmic discharge is selected. Main plasma parameters of the discharge: the toroidal magnetic field is 1.3T, the plasma current is $150 \mathrm{kA}$, the line averaged electron density is $0.8 \times 10^{19} \mathrm{~m}^{-3}$. Two $f_{D}$ curves in Fig. $2 b$ show the small difference mainly caused by the different equivalent sampling frequencies. The phase derivative method costs less calculation time than that of the FFT based CoG method. However, the difference does not affect the analysis of $E_{r}$ fluctuation. As shown in Fig.2c are the cross-power spectra of two poloidal Doppler signals with same launching frequency. The poloidal separation is about $30 \mathrm{~cm}$. There are artificial higher frequency components $(30-50 \mathrm{kHz})$ in the spectrum from phase derivative method, which could be induced by the zero order of the reflected signal as discussed in Ref. [16]. A coherent peak of $12 \mathrm{kHz}$ is observed from the spectra. The mode frequency is close to the geodesic acoustic frequency $\omega_{G A M}=\sqrt{2\left(T_{e}+7 T_{i} / 4\right) / M_{i}} / R$, suggesting that it is a GAM oscillation.

\section{Characteristics of GAM}

\subsection{Spatial structure}

With poloidally and toroidally equipped Doppler reflectometers in HL-2A, the long range correlation measurements for $E_{r}$ can be realized. Figure $3 \mathrm{a}$ and $3 \mathrm{~b}$ shows the poloidal and toroidal 

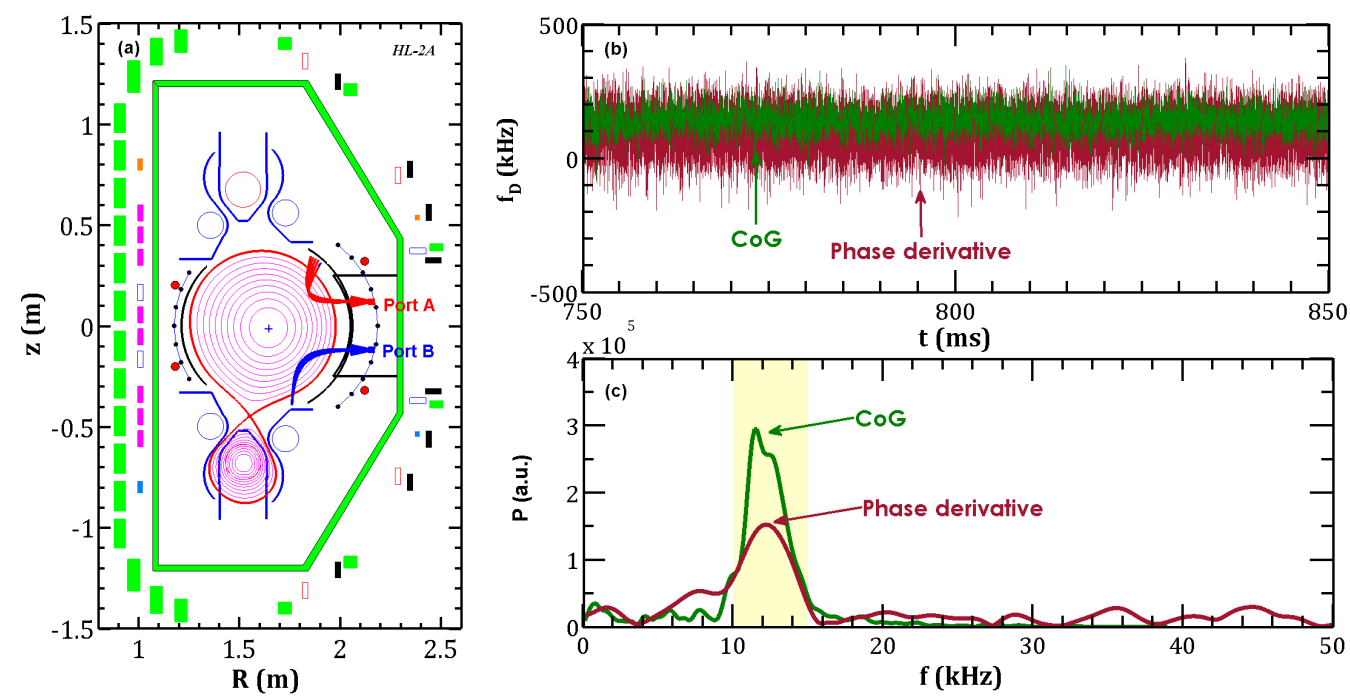

Figure 2: (a) Poloidal arrangement of Doppler reflectometers in the HL-2A tokamak. Two ports (A and B) are used for X-mode measurements in this paper, (b) time traces of the $f_{D}$ estimated by CoG and phase derivative methods, (c) cross-power spectra of the $f_{D}$ signals between two poloidally separated ports.
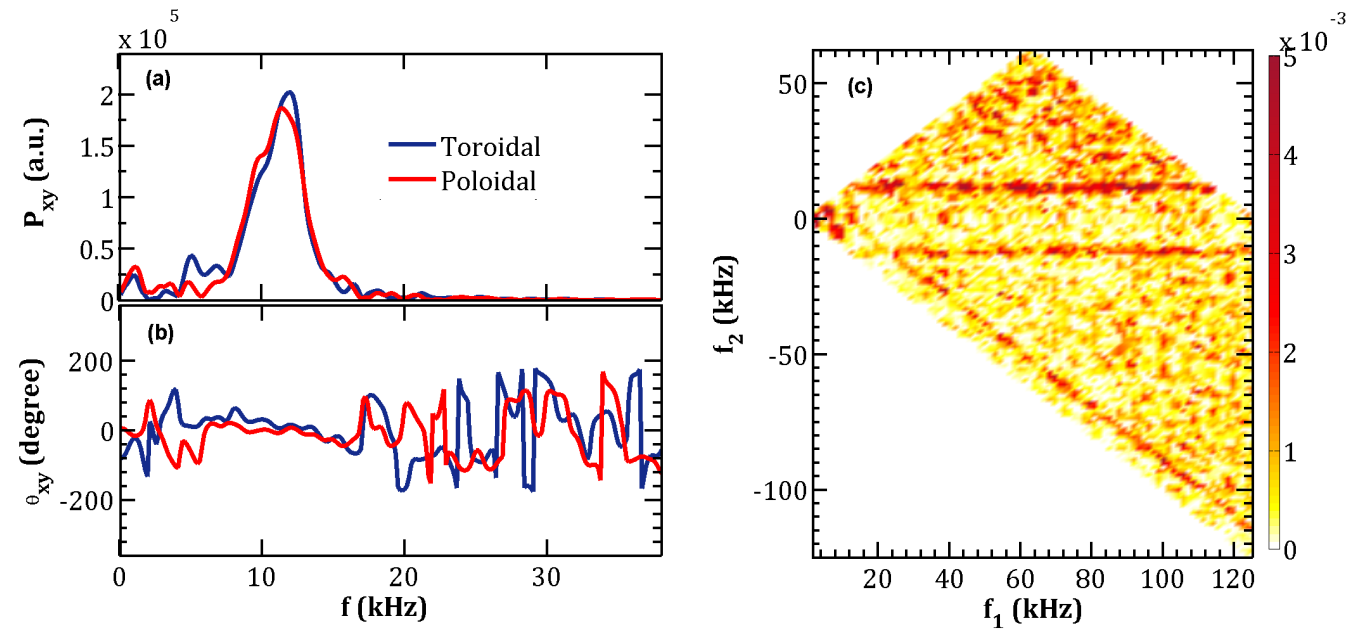

Figure 3: (a) Poloidal and toroidal cross-power spectra of $E_{r}$ fluctuations between two separated ports , (b) and corresponding cross-phase spectra, (c) squared bi-coherence spectrum of the $f_{D}$ fluctuations plotted in the $f_{1}-f_{2}$ plane.

cross-power and cross-phase spectra of $E_{r}$ fluctuations. The distance between two poloidally separated ports is about $30 \mathrm{~cm}$. In toroidal direction, the distance is about $150 \mathrm{~cm}$. Two cross-power spectra in Fig. 3a show clear coherent peaks $(f \simeq 12 \mathrm{kHz})$. Corresponding to the frequencies of the peaks, the phase shifts (Fig.3b) in both poloidal and toroidal directions are near zero, demonstrating the two dimensional symmetry $(m=0$ and $n=0)$ of GAM appeared in the fluctuation component of $E_{r}$. Figure $3 \mathrm{c}$ is the squared auto-bicoherence of the $f_{D}$ fluctuations. It is calculated by $b^{2}\left(f_{1}, f_{2}\right)=\frac{\left\langle\left\langle\left.\hat{X}\left(f_{1}\right) \hat{X}\left(f_{2}\right) \hat{X}^{*}\left(f_{1}+f_{2}\right)\right|^{2}\right\rangle\right.}{\left\langle\left|\hat{X}\left(f_{1}\right) \hat{X}\left(f_{2}\right)\right|^{2}\right\rangle\left\langle\left.\hat{X}\left(f_{1}+f_{2}\right)\right|^{2}\right\rangle}$, where $\left.X \hat{(} f\right)$ is the Fourier transform of the $f_{D}$ and $\hat{X}^{*}(f)$ 


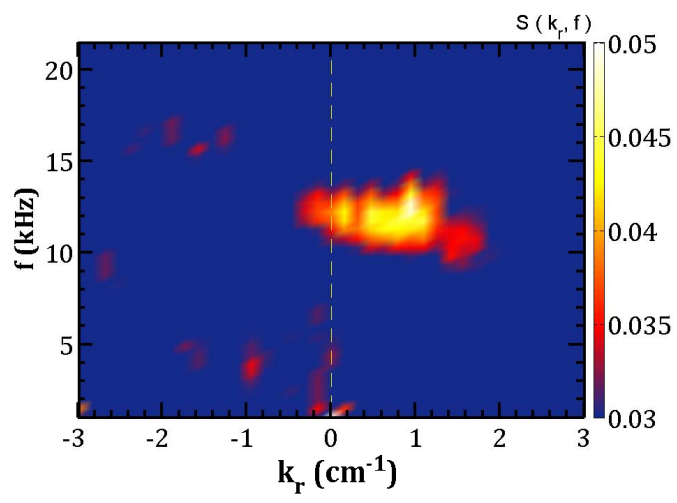

Figure 4: Radial wave-number spectrum of $E_{r}$ fluctuations in edge plasma.

is the complex conjugate. The angular bracket represents the ensemble average. The squared bicoherence $b^{2}\left(f_{1}, f_{2}\right)$ is an indicator for the strength of nonlinear coupling of three wave at $f_{1}, f_{2}$ and $f_{1}+f_{2}$ [17]. There are three noticeable peaks with higher values of $b^{2}\left(f_{1}, f_{2}\right)$ are shown in Fig. $3 \mathrm{c}$, the frequency $f_{1}+f_{2}=12 \mathrm{kHz}$ and $f_{2}= \pm 12 \mathrm{kHz}$ is corresponding to GAM frequency in Fig. 3a, suggesting that the nonlinear interaction is responsible for the generation of the GAM.

In additional to the symmetry property of GAM structure in both poloidal and toroidal directions, its radial characteristics are also described by radially separated measurements of multichannel Doppler reflectometers. Figure 4 shows the wave-number spectrogram $S\left(k_{r}, f\right)$ obtained from $36 \mathrm{GHz}$ and $38 \mathrm{GHz}$ channel of the Doppler reflectometry. The radial separation is $1 \mathrm{~cm}$. A noticeable peak, which corresponds to the GAM, is clearly observed. The spectral-averaged wavenumber can be estimated by $k_{r}\left(f=f_{G A M}\right)=\sum_{k_{r}} k_{r} \cdot S\left(k_{r} \mid f=f_{G A M}\right)$, here the $S\left(k_{r} \mid f=f_{G A M}\right)$ is the conditional wave-number spectral density. For the GAM in Fig. $4, k_{r}$ is $0.68 \mathrm{~cm}^{-1}$. The positive value of the wave-number indicates a radial outward phase velocity of the GAM.

\subsection{Collisional damping}

Theoretical work predicted that the GAM would be strongly damped at high collisional plasmas. The collisional damping rate of GAM is proportional to the ion-ion collisional frequency [18]. Experimentally, the collisionality can be increased by increasing the ion density and /or decreasing the ion temperature. This requirement is met by plasma fueling, such as Fig. 5a shown. The Doppler measurement is about $4 \mathrm{~cm}$ inside the LCFS. Owing to the X-mode polarization, the radial movement of the measurement position is less than $1 \mathrm{~cm}$. Figure $5 \mathrm{~b}$ is the Doppler spectrogram. Preliminary, both the turbulence poloidal velocity and intensity is modulated by the gas puff. Figure $5 \mathrm{c}$ is the spectrogram of $E_{r}$ fluctuations. The noticeable mode is the GAM. Obviously, the GAM amplitude (Fig.5d) is also modulated by gas puff. The fueling increases the collisionality.Then the GAM amplitude is reduced. In return, the stronger GAM is accompanied by the decreased collisionality. The result reveals that the collisional damping is responsible for the decrease of the GAM amplitude. The modulated GAM amplitude is inversely correlated with the turbulence intensity as Fig. 5e shown, suggesting that the GAM has the regulating effect on turbulence. 


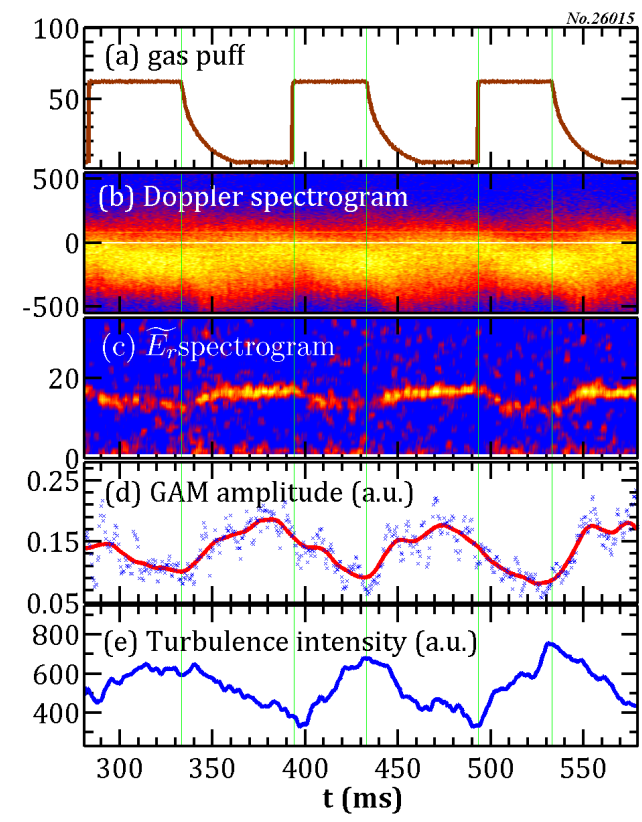

Figure 5: Collisonal damping for GAM. (a) gas puff monitor, (b) Doppler spectrogram, (c) spectrogram of $E_{r}$ fluctuations, (d) GAM amplitude and (e) turbulence intensity obtained from Doppler reflectometry.

\section{Conclusion}

Recently, several correlation Doppler reflectometers have been newly developed in HL-2A. The multi-channel systems allow studying radial properties of edge turbulence and its long range correlations in both poloidal and toroidal directions, simultaneously. Specifically, the non disturbing diagnostic is used to routinely measure the perpendicular rotation velocity of turbulence and the density fluctuations. For the extraction of $E_{r}$ fluctuations from Doppler reflectometry signal, two data processing methods ( $\mathrm{CoG}$ and phase derivative) are introduced. With correlation Doppler reflectometers, three dimensional spatial structure of GAM is characterized, including the symmetry feature of poloidal and toroidal $E_{r}$ fluctuations, and the radial propagation of GAM. The bicoherence analysis suggests that the three wave nonlinear interaction would be the mechanism for the generation of GAM. The temporal behaviors of GAM during gas puff modulation experiments are investigated, which suggests that the collisional damping plays a role in suppressing the GAM oscillation level, and hence, weakening the regulating effects of GAM on ambient turbulence.

\section{Acknowledgments}

The authors would like to gratefully acknowledge the HL-2A team for the support of the experiments. This work is partially supported by Natural Science Foundation of China under Grant Nos. 10990213, 11305053,11475057 and 11375057, and partially supported by Chinese National Fusion Project for ITER under Grant Nos. 2013 GB107000 and 2014GB108000. 


\section{References}

[1] Zou X.L. et al Proc. 26th EPS Conf. on Contr. Fusion and Plasma Physics (Maastricht) 1999 ECA vol 23J 1041

[2] Hirsch M. et al 2001 Plasma Phys. Control. Fusion 431641

[3] Conway G.D. et al 2004 Plasma Phys. Control. Fusion 46951

[4] Rosenbluth M. N. and Hinton F. L. 1998 Phys. Rev. Lett. 80724

[5] Lin Z. et al 1998 Science 2811835

[6] Horton W. 1999 Rev. Mod. Phys. 71735

[7] Conway G.D. et al 2005 Plasma Phys. Control. Fusion 471165

[8] Conway G.D. et al 2011 Phys. Rev. Lett. 106065001

[9] Vermare L. et al 2012 Nucl. Fusion 52063008

[10] Gurchenko A.D. 2013 Plasma Phys. Control. Fusion 55085017

[11] Kramer-Flecken A. et al 2006 Phys. Rev. Lett. 97045006

[12] Shi. Z.B. et al 2015 submitted to Rev. Sci. Instrum.

[13] Duan X.R. et al 2013 Nucl. Fusion 53104009

[14] Shi. Z.B. et al 2015 The 12th International Reflectometry Workshop, Juelich, Germany

[15] Zhong W.L. et al 2014 Rev. Sci. Instrum. 85013507

[16] Holzhauer E. et al 1998 Plasma Phys. Control. Fusion 401869

[17] Kim Y.C. et al 1979 IEEE Trans. Plasma Sci. 7120

[18] Gao Z. 2013 Phys. Plasmas 20032501 